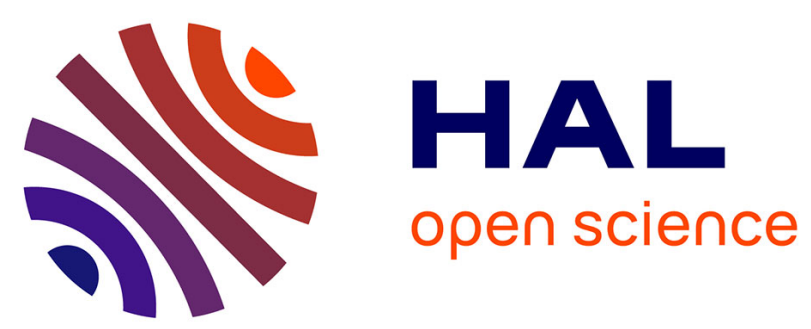

\title{
Observations of the Interplanetary Hydrogen during Solar Cycles 23 and 24. What can We Deduce about the Local Interstellar Medium?
}

Frédéric Vincent, Olga Katushkina, Lotfi Ben-Jaffel, Walter M. Harris, Vladislav Izmodenov, Eric Quémerais, Dimitra Koutroumpa, John Clarke

\section{To cite this version:}

Frédéric Vincent, Olga Katushkina, Lotfi Ben-Jaffel, Walter M. Harris, Vladislav Izmodenov, et al.. Observations of the Interplanetary Hydrogen during Solar Cycles 23 and 24. What can We Deduce about the Local Interstellar Medium?. The Astrophysical journal letters, 2014, 788 (2), pp.L25. 10.1088/2041-8205/788/2/L25 . hal-00998272

\section{HAL Id: hal-00998272 \\ https://hal.science/hal-00998272}

Submitted on 17 Jun 2014

HAL is a multi-disciplinary open access archive for the deposit and dissemination of scientific research documents, whether they are published or not. The documents may come from teaching and research institutions in France or abroad, or from public or private research centers.
L'archive ouverte pluridisciplinaire HAL, est destinée au dépôt et à la diffusion de documents scientifiques de niveau recherche, publiés ou non, émanant des établissements d'enseignement et de recherche français ou étrangers, des laboratoires publics ou privés. 


\title{
OBSERVATIONS OF THE INTERPLANETARY HYDROGEN DURING SOLAR CYCLES 23 AND 24. WHAT CAN WE DEDUCE ABOUT THE LOCAL INTERSTELLAR MEDIUM?
}

\author{
Frédéric E. Vincent ${ }^{1,2}$, Olga Katushinan ${ }^{3}$, LOtFi Ben-JafFel ${ }^{2}$, WAlter M. Harris ${ }^{4}$, Vladislav IzMOdenov ${ }^{3,5,6}$, \\ Eric Quémerais ${ }^{1}$, Dimitra Koutroumpa ${ }^{1}$, and John Clarke ${ }^{7}$ \\ ${ }^{1}$ Université Versailles St.-Quentin, Sorbonne Universités, UPMC Univ. Paris 06, CRNS/INSU, LATMOS-IPSL, \\ 11 boulevard d'Alembert, 78280 Guyancourt, France \\ 2 UPMC Univ. Paris 06, UMR7095, Institut d'Astrophysique de Paris, F-75014, Paris, France \\ ${ }^{3}$ Space Research Institute of Russian Academy of Sciences, Moscow, Russia \\ ${ }^{4}$ University of Arizona, Lunar and Planetary Laboratory, 1629 E. University Blvd., Tucson, AZ 85721, USA \\ ${ }^{5}$ Department of Mechanics and Mathematics, Lomonosov Moscow State University, Moscow, Russia \\ ${ }^{6}$ Institute for Problems in Mechanics of Russian Academy of Sciences, Moscow, Russia \\ ${ }^{7}$ Center for Space Physics, Boston University, 725 Commonwealth Avenue, Boston, MA 02215, USA \\ Received 2014 January 31; accepted 2014 April 10; published 2014 May 30
}

\begin{abstract}
Observations of interstellar helium atoms by the Interstellar Boundary Explorer (IBEX) spacecraft in 2009 reported a local interstellar medium (LISM) velocity vector different from the results of the Ulysses spacecraft between 1991 and 2002. The interplanetary hydrogen (IPH), a population of neutrals that fills the space between planets inside the heliosphere, carries the signatures of the LISM and its interaction with the solar wind. More than $40 \mathrm{yr}$ of space-based studies of the backscattered solar Ly $\alpha$ emission from the IPH provided limited access to the velocity distribution, with the first temporal evolution map of the IPH line-shift during solar cycle 23. This work presents the results of the latest IPH observations made by the Hubble Space Telescope's Space Telescope Imaging Spectrograph during solar cycle 24 . These results have been compiled with previous measurements, including data from the Solar Wind Anisotropies instrument on the Solar and Heliospheric Observatory. The whole set has been compared to physically realistic models to test both sets of LISM physical parameters as measured by Ulysses and IBEX, respectively. This comparison shows that the LISM velocity vector has not changed significantly since Ulysses measurements.
\end{abstract}

Key words: local interstellar matter - solar neighborhood - solar wind - Sun: activity - Sun: heliosphere Sun: UV radiation

Online-only material: color figures

\section{INTRODUCTION}

The Sun probes its galactic surroundings as it flows through the local interstellar medium (LISM), also called the local interstellar cloud, a diffuse warm and partially ionized medium, mainly composed of atomic hydrogen (Frisch et al. 2011). The relative motion of the LISM with respect to the Sun creates an interstellar wind that interacts with the supersonic expanding solar wind (SW). Because the ionized component of the LISM and the SW are both magnetized plasmas, they cannot penetrate each other, so the SW carves out a cavity called the heliosphere (Parker 1961; Baranov et al. 1971; Axford 1972). While the ionized component of the LISM is slowed down and deflected at the boundary of the heliosphere (the heliopause), interstellar neutral atoms can cross the heliospheric interface (Blum \& Fahr 1970). Within the heliosphere, hydrogen atoms form a population called interplanetary hydrogen (IPH) that has been observed through the backscattering of solar Ly $\alpha$ photons from the 1970s (Bertaux \& Blamont 1971; Thomas \& Krassa 1971) until now. While crossing the heliospheric interface, the bulk motion of interstellar hydrogen atoms is slowed down because of resonance charge exchange reactions with the slowing LISM protons (Wallis 1975; Baranov \& Malama 1993; Linsky \& Wood 1996). Inside the heliosphere, the IPH is affected by several temporally dependent processes related to the SW and the solar Ly $\alpha$ radiation, resulting in variations of the IPH bulk velocity with solar cycle (Quémerais et al. 2006a; Izmodenov et al. 2008; Vincent et al. 2011).
Interstellar helium atoms are almost unaffected by the heliospheric interface and carry a more pristine signature of the LISM. Observations of helium atoms by Ulysses between 1992 and 2001 yielded a LISM velocity of about $26.3 \pm 0.4 \mathrm{~km} \mathrm{~s}^{-1}$ (Witte 2004), while more recent helium observations by the Interstellar Boundary Explorer (IBEX) in 2009 reported a lower velocity of $23.2 \pm 0.3 \mathrm{~km} \mathrm{~s}^{-1}$ (McComas et al. 2012); these variations raise the question of a possible deceleration of the LISM relatively to the heliosphere. On a larger scale, the LISM velocity can also be estimated by Doppler triangulation from absorption measurements over long distances toward nearby stars (within $100 \mathrm{pc}$ of the Sun): Bertin et al. (1993) obtained a velocity of $25.5 \pm 1.0 \mathrm{~km} \mathrm{~s}^{-1}$ during the Ulysses era, while more recent measurements provided a lower value of $23.84 \pm 0.9 \mathrm{~km} \mathrm{~s}^{-1}$ (Redfield \& Linksy 2008).

The heliosphere is also affected by the local interstellar magnetic field (LIMF). Models taking into account the obliquity of the LIMF showed a severe distortion of the heliosphere (Fahr et al. 1988; Ratkiewicz et al. 1998; Pogorelov \& Matsuda 1998). These predictions were confirmed by a multi-observational approach: the deviation of the intensity maximum in the Ly $\alpha$ flux from the upwind direction as detected by Voyager 1/ Ultraviolet Spectrometer (Ben-Jaffel et al. 2000), the deflection of the interstellar hydrogen flow with respect to the helium flow as observed by Solar and Heliospheric Observatory (SOHO)/ Solar Wind ANisotropy (SWAN; Lallement et al. 2005, 2010; Izmodenov et al. 2005), the difference in the termination shock heliospheric distances detected by the Voyager 1 and Voyager 2 
spacecraft (Ratkiewicz \& Grygorczuk 2008; Izmodenov 2009; Pogorelov et al. 2009; Opher et al. 2009), and the ribbon of energetic neutral atoms around the heliosphere discovered by Cassini (Krimigis et al. 2009) and IBEX (McComas et al. 2009).

As the IPH velocity depends on the relative motion of the LISM with respect to the heliosphere, we propose a complementary approach to studying the LISM. In this Letter, we describe IPH observations made in 2012 and 2013 by the Hubble Space Telescope (HST)/Space Telescope Imaging Spectrograph (STIS). We compile these with other IPH observations, and compare them with a physically realistic model in order to provide constraints on the LISM parameters and discuss the discrepancy between LISM velocity measurements from Ulysses and IBEX.

\section{OBSERVATIONS OF THE INTERPLANETARY HYDROGEN}

\subsection{Description of the Instrument and the Signal}

Servicing on the HST since 1997, STIS is an ultraviolet spectrograph, including an echelle mode that combines an echelle grating with a cross-disperser grating (Woodgate et al. 1998).

STIS has been used to observe the IPH along a line of sight (LOS; $\lambda=253.2$ and $\beta=7.0$ in ecliptic coordinates) that is near the upwind direction, on 2001 March 29, 2012 April 9, and 2013 April 22. The velocity of the Earth along the LOS was, respectively, $27.0 \mathrm{~km} \mathrm{~s}^{-1}, 23.9 \mathrm{~km} \mathrm{~s}^{-1}$, and $19.6 \mathrm{~km} \mathrm{~s}^{-1}$. These observations used the high-resolution echelle grating $\mathrm{E} 140 \mathrm{H}$ with the long slit $52^{\prime \prime} \times 0.5$ and the FUV-MAMA detectors, the Doppler shift due to HST motion was corrected by the STIS pipeline. The observations were processed to subtract the counts due to the dark current, flat-field the spectro-image, and correct the distortions due to a vertical misalignment and the variation of sensitivity curves along the dispersion correction (Walsh et al. 2001; Hernandez et al. 2014).

Using a long slit allows the collection of more photons and an increase of the signal to noise from an extended source such as IPH, but this configuration results in the superposition of different orders of the echelle spectrum, especially the contamination of the Ly $\alpha$ line $(121.6 \mathrm{~nm})$ by the $130.4 \mathrm{~nm}$ triplet line of geocoronal oxygen (Vincent et al. 2011).

\subsection{Data Analysis}

The background light was fitted by a two-degree polynomial and was then subtracted from the signal. To subtract the contamination by the geocoronal oxygen, we used the signal at the location of the occulting bars where the Ly $\alpha$ line is blocked. This signal has been smoothed by three to obtain a similar signal-to-noise ratio, compared to the main signal outside of the occulting bars.

The line-spread function (LSF) was not available because STIS observations were done in an unsupported mode. Hence we constructed an ad hoc LSF from the convolution of a previously measured LSF from a supported mode with a square filter having the same width as the slit used for the observations (see the second paragraph of Section 2.3 in Vincent et al. 2011).

We fitted separately the blue side and the red side of the geocorona line profile, using STIS observations made in 2000 and 2001, respectively, as the corresponding side was not overlapped by the IPH or any O I contamination because of the geometry of the observations (see the third paragraph of Section 2.3 in Vincent et al. 2011). Each side was fitted by the convolution of the ad hoc LSF with a sum of two Voigt functions that fit the core and the wing of that side.

We fitted the IPH line profile with the convolution of the LSF and a Voigt function. The free parameters were the IPH line profile and separation from the geocorona, all of them were fitted simultaneously by solving the least-squares problem with an algorithm implementing the Levenberg-Marquardt technique (Markwardt 2009). We derived the IPH line-shift from the Doppler shift between the IPH and the geocorona line centers, and after subtraction of the Earth's velocity along the LOS. Radiative transfer computations showed that any observed lineshift represents approximately the bulk velocity of the IPH atoms, projected and integrated along the LOS from the Earth (Quémerais 2000). We computed the $1 \sigma$ errors as the quadratic sum of the instrumental and statistical uncertainties (for more details, see the fifth paragraph of Section 2.3 in Vincent et al. 2011).

For the 2001 observations, this present work yielded a value of $22.6 \pm 0.5 \mathrm{~km} \mathrm{~s}^{-1}$, consistent with a previous analysis that was fitting all spectral features (instead of removing the signal from the occulting bars) and gave a result of $22.4 \pm 0.4 \mathrm{~km} \mathrm{~s}^{-1}$ (Vincent et al. 2011). The similarity between the results from different methods on the same data set validates the robustness of the method that has been used for this data analysis. Our best fits to the other STIS observations provide a line shift of $23.5 \pm$ $0.5 \mathrm{~km} \mathrm{~s}^{-1}$ in 2012, and $23.3 \pm 0.5 \mathrm{~km} \mathrm{~s}^{-1}$ in 2013, as shown respectively in Figures 1 and 2.

\section{MODEL OF THE INTERSTELLAR HYDROGEN DISTRIBUTION IN THE HELIOSPHERE}

To interpret the data, we use an advanced three-dimensional (3D) non-stationary kinetic model of the interstellar hydrogen distribution inside the heliosphere (Izmodenov et al. 2013). This model combines the relative numerical simplicity of the classical hot-type models (e.g., Bzowski et al. 1997) with the possibility to take into account local 3D and time-dependent effects as well as the disturbances of the interstellar atoms distribution at the heliospheric boundaries. In order to calculate spatial and velocity distribution of the IPH near the Sun as well as the backscattering of solar Ly $\alpha$ photons by the IPH, we followed three separate steps.

\subsection{Outer Heliosphere}

At the first step, we run a global (axisymmetric or 3D) stationary kinetic-gasdynamical model of the interaction between the SW and the LISM, finding the global structure of the heliospheric interface region as well as parameters of the interstellar hydrogen distribution far away from the Sun (Baranov \& Malama 1993; Izmodenov et al. 2009, 2013). Because interstellar hydrogen atoms have large mean free paths comparable with the characteristic distance of the heliosphere (hundreds of $\mathrm{AU})$, the evolution of the velocity distribution must be described by the kinetic Boltzmann equation. The model computes the perturbations of the hydrogen velocity distribution function due to charge exchange in the LISM/SW interaction region. These perturbations provide the non-Maxwellian velocity distribution function at $90 \mathrm{AU}$ from the Sun (Katushkina \& Izmodenov 2010, 2011).

\subsection{Inner Heliosphere}

At the second step, we use the obtained hydrogen distribution at $90 \mathrm{AU}$ as the boundary conditions for our 3D time-dependent 

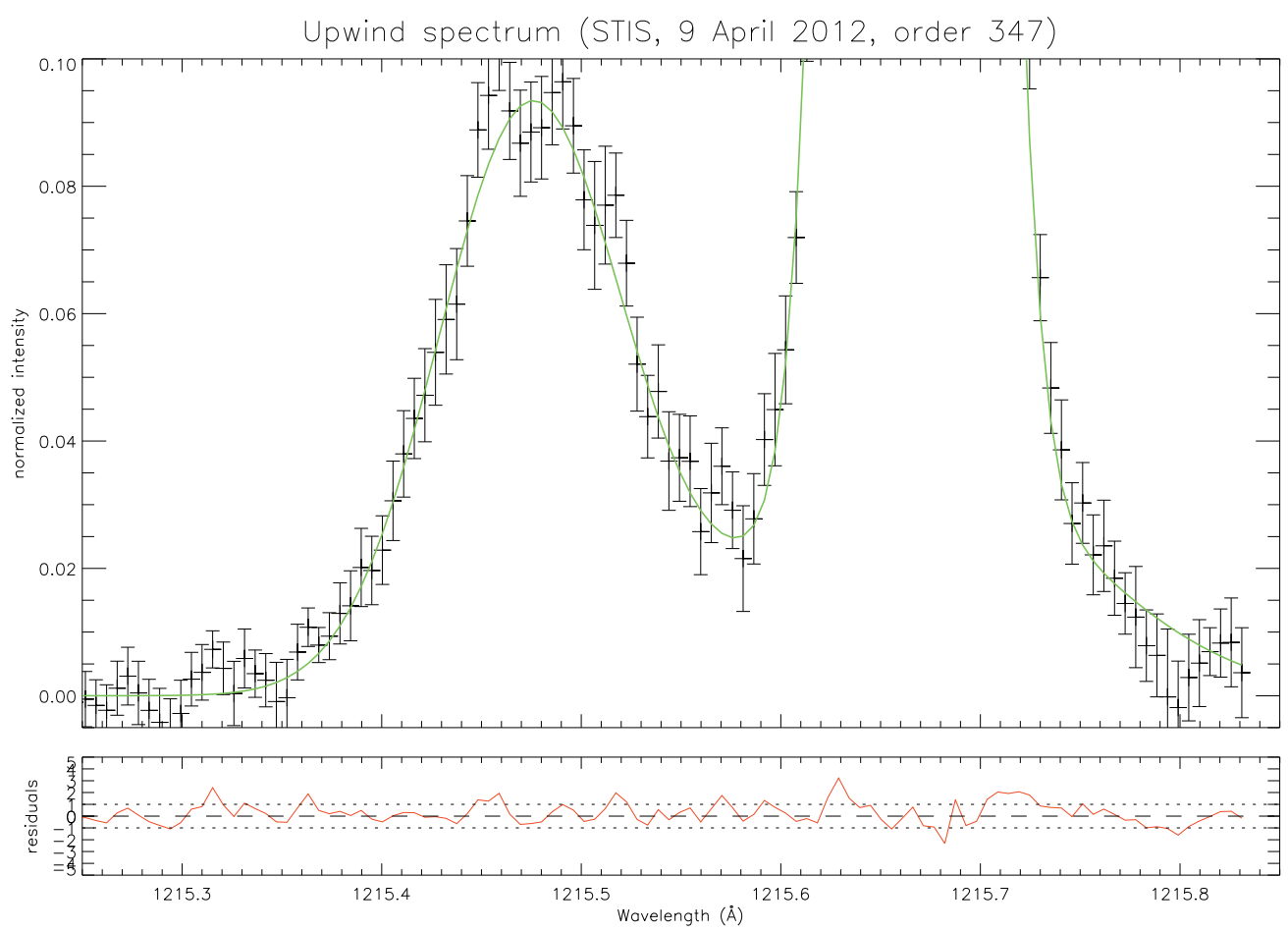

Figure 1. STIS observations in 2012. The best fit gives a line-shift of $23.5 \pm 0.5 \mathrm{~km} \mathrm{~s}^{-1}$ in the heliospheric reference frame. (A color version of this figure is available in the online journal.)
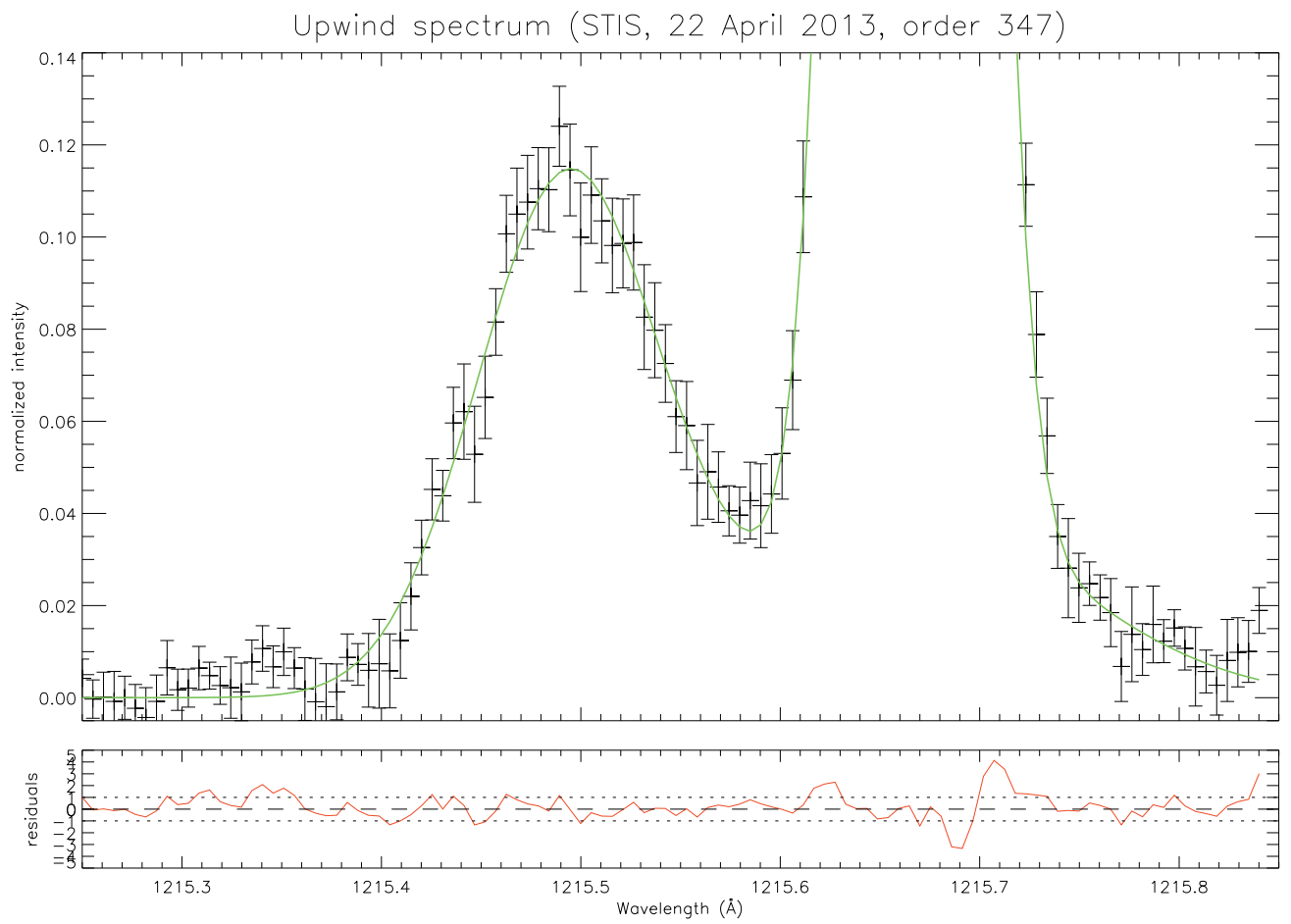

Figure 2. STIS observations in 2013. The best fit gives an IPH line-shift of $23.3 \pm 0.5 \mathrm{~km} \mathrm{~s}^{-1}$ in the heliospheric reference frame.

(A color version of this figure is available in the online journal.)

kinetic model of hydrogen distribution in the heliosphere and find the detailed hydrogen velocity distribution function near the Sun (for details, see Izmodenov et al. 2013). Inside the heliosphere, the IPH atoms are subject to counteracting forces: the Sun gravity and the solar radiation pressure, whose ratio yields the dimensionless balanced parameter $\mu$. In the 3D timedependent case, this parameter $\mu$ depends on time, heliolatitude and individual radial velocity of $\mathrm{H}$ atoms. Two ionization processes lead to the loss of $\mathrm{H}$ atoms: charge exchange with the SW protons $\left(\beta_{e x}\right)$ and photoionization $\left(\beta_{\mathrm{ph}}\right)$. The model assumes that charge exchange and photoionization rates are proportional to the fluxes of the SW protons and solar photons, respectively. The model assumes that the ionization rates decrease as $1 / r^{2}$, so they can be deduced from values at Earth's orbit $\left(\beta_{\mathrm{ex}, E}\right.$ and $\beta_{\mathrm{ph}, E}$ ). Figure 3 represents the in-ecliptic values of the parameters $\mu, \beta_{\mathrm{ex}, E}$, and $\beta_{\mathrm{ph}, E}$ that are given, respectively, by the 

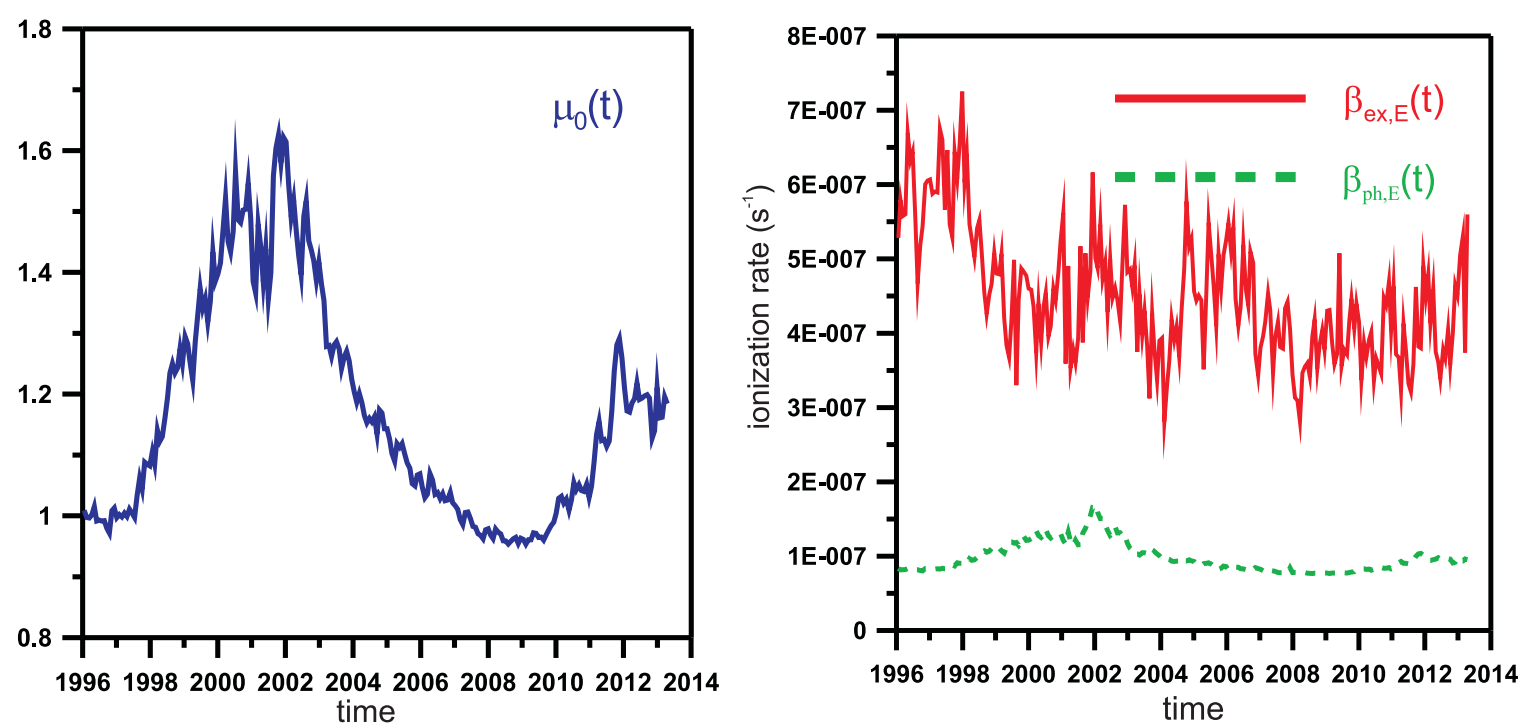

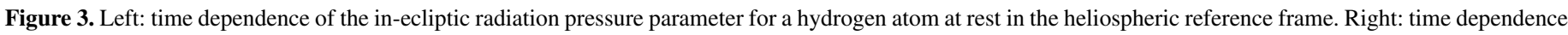
of in-ecliptic ionization rates at Earth's orbit (charge exchange: $\beta_{\mathrm{ex}, E}$; photoionization: $\beta_{\mathrm{ph}, E}$ ).

(A color version of this figure is available in the online journal.)

LASP Interactive Solar Irradiance Center, the OMNI-2 database, and the SOLAR2000 database. The heliolatitudinal dependance of these ionization rates is given from the analysis of $\mathrm{SOHO} /$ SWAN data (Quémerais et al. 2006b).

\subsection{Radiative Transfer}

Lastly, the described hydrogen distributions in the heliosphere have been used to calculate the line shift of the backscattered solar Ly $\alpha$ radiation, as measured at the Earth's orbit. We used a radiative transfer model based on the simplified self-absorption approach (Quémerais \& Izmodenov 2002): it takes into account only singly scattered photons but includes extinction along the LOS between the scattering point and the observer. The backscattered solar Ly $\alpha$ line shifts have been calculated toward the direction of 8.9 of ecliptic latitude, and 252.2 of ecliptic longitude. This direction corresponds to the averaged direction of hydrogen flow inside the heliosphere obtained in Lallement et al. (2010) from the $\mathrm{SOHO/SWAN} \mathrm{data.} \mathrm{Calcula-}$ tions were performed for an observer at the Earth orbit as it would be on April 1 (i.e., the ecliptic longitude of the observer is $191^{\circ}$ ) for all years from 1996 to 2013 .

\subsection{LISM parameters}

At the first step that describes the SW/LISM interaction, we consider numerical models with four different sets of the LISM boundary conditions (see Table 1), using Ulysses or IBEX results for the velocity vector of the LISM, including an interstellar magnetic field (IsMF) or not. For all models, the number densities of protons and hydrogen atoms in the LISM are $n_{p, \text { LISM }}=0.06 \mathrm{~cm}^{-3}, n_{\mathrm{H}, \mathrm{LISM}}=0.18 \mathrm{~cm}^{-3}$; the temperature of the LISM is $T_{\mathrm{LISM}}=6530 \mathrm{~K}$, within the range provided by Ulysses/GAS data, yielding a Mach number of about 2.

In cases $A$ and $B$, the relative $S W /$ LISM velocity is $\left|\mathbf{V}_{\text {LISM }}\right|=$ $26.4 \mathrm{~km} \mathrm{~s}^{-1}$, and its direction in $\mathrm{J} 2000$ ecliptic coordinates has the longitude $\lambda_{\text {LISM }}=75.4$ and the latitude $\beta_{\text {LISM }}=-5.2$, as reported by the analysis of Ulysses/GAS data on the interstellar helium fluxes (Witte 2004). In cases C and D, the relative $\mathrm{SW} / \mathrm{LISM}$ velocity vector was taken from the analysis of the interstellar helium fluxes measured by IBEX-Lo in 2009-2010
Table 1

Parameters of the Local Interstellar Medium (LISM) and the Local Interstellar Magnetic Field (LIMF) for the Different Models

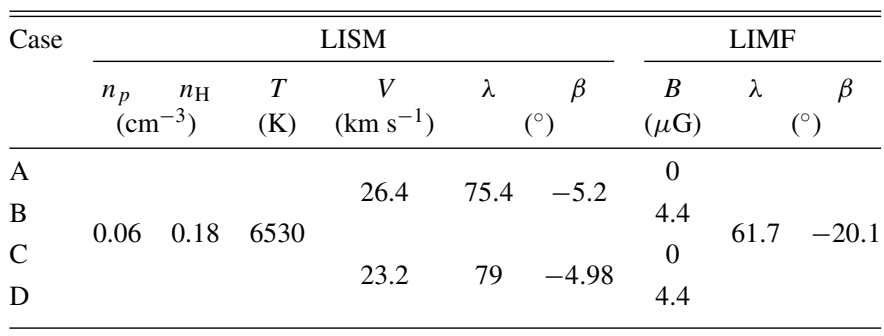

Notes. Models A and B use the parameters derived by Ulysses, while Models C and D use the results from IBEX. Models $\mathrm{C}$ and D introduce a $4.4 \mu \mathrm{G}$ magnetic field that lies within the hydrogen deflection plane and makes a $20^{\circ}$ angle with the LISM velocity vector.

(McComas et al. 2012): thus a direction of $\left(\lambda_{\text {LISM }}=79^{\circ}\right.$, $\left.\beta_{\text {LISM }}=-4.98\right)$ with a speed of $\left|\mathbf{V}_{\text {LISM }}\right|=23.2 \mathrm{~km} \mathrm{~s}^{-1}$.

In cases $\mathrm{A}$ and $\mathrm{C}$, hydrogen distribution at $90 \mathrm{AU}$ is taken from the results of a stationary axisymmetric self-consistent kineticgasdynamical model of the heliospheric interface developed by Baranov \& Malama (1993), where no magnetic fields were taken into account. Cases B and D employ the results of a 3D kineticMHD model of the heliospheric interface (Izmodenov et al. $2005,2009)$ that includes the IsMF, assuming an intensity of $4.4 \mu G$. The IsMF vector lies within the so-called hydrogen deflection plane defined by Lallement et al. (2010) and is assumed to make an angle of $20^{\circ}$ with the LISM velocity vector, this angle giving the best fit of the model for the distances of the termination shock as detected by Voyager (Izmodenov et al. 2009).

\section{DISCUSSION}

\subsection{Data from the SWAN Instrument}

Interplanetary Ly $\alpha$ line profiles were also derived from the data obtained with the $\mathrm{SOHO} / \mathrm{SWAN}$ hydrogen absorption cell that yields annually averaged observations from weekly fullsky maps. The SWAN results cover a waning phase of the solar 


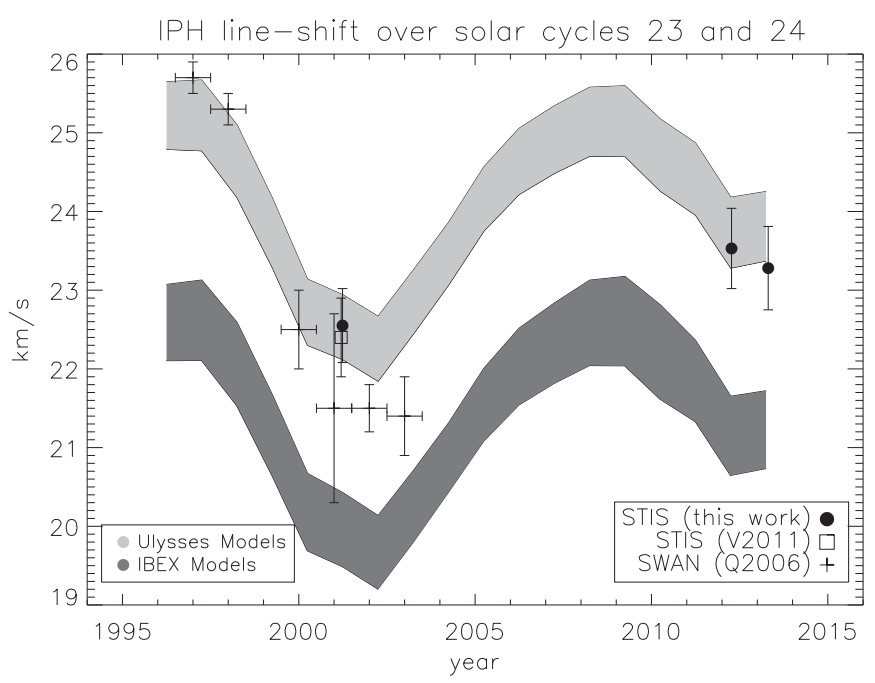

Figure 4. Interplanetary hydrogen line-shift in the upwind direction over solar cycles 23 and 24, including STIS results reported by this work (filled circles) and a previous analysis (squares; Vincent et al. 2011), and SWAN results (crosses; Quémerais et al. 2006a). Results from numerical models made by the Moscow group (Izmodenov et al. 2013) have been overplotted: the dim and the dark bands represent models using Ulysses and IBEX results, respectively, for the LISM velocity vector; in both cases, the lower limit represent a model with no interstellar magnetic field (IsMF), while the upper limit includes an $4.4 \mu \mathrm{G}$ IsMF that belongs to the hydrogen deflection plane and makes a $20^{\circ}$ angle with the LISM velocity vector.

cycle, and indicate a velocity decrease from $25.7 \pm 0.2 \mathrm{~km} \mathrm{~s}^{-1}$ to $21.4 \pm 0.5 \mathrm{~km} \mathrm{~s}^{-1}$ between 1997 and 2003 (Quémerais et al. 2006a). With the STIS observations, the data set covers a significant fraction of the previous and current solar cycles (23 and 24).

\subsection{Comparison with Models}

Figure 4 shows the IPH velocities obtained from observations by SWAN and STIS. In order to show the solar cycle effect and the influence of LISM, we overplotted the predictions from the four numerical models that are described in the previous section and whose parameters are summed up in Table 1. The shift between different models remains almost constant over the solar cycle: introducing a LIMF induces a velocity increase of about $0.9 \mathrm{~km} \mathrm{~s}^{-1}$; in the absence of the LIMF, the interstellar flow is supersonic and undergoes a larger deceleration at the bow shock, resulting in a lower IPH velocity.

Globally, IPH data fit Ulysses-based models better than IBEXbased models, especially for STIS observations (2001, 2012, 2013) and for a fraction of SWAN observations (1996, 1997, 2000). The set of equations used to derive the LISM velocity from IBEX data allows a bounding range whose upper value is $25.7 \mathrm{~km} \mathrm{~s}^{-1}$ (McComas et al. 2012), so very close to the value measured by Ulysses, which could resolve the current discrepancy between IBEX and Ulysses data. Moreover, a sensitivity study of the LIMF, fitting Voyager in situ plasma measurements and IBEX energetic neutral atoms ribbon, supports Ulysses results as well (Ben-Jaffel et al. 2013).

Among the two Ulysses-based models, IPH data is better fitted by the model that does not include any LIMF. As the LIMF has been measured through a multi-observational approach (e.g., Lallement et al. 2005; Izmodenov et al. 2005; Ratkiewicz \& Grygorczuk 2008; Izmodenov 2009; McComas et al. 2009), this discrepancy suggests an unidentified weakness in the model.

\subsection{Perspectives}

Some discrepancies between data and models show that an additional modeling effort is necessary to interpret the observations and reach a better understanding of the heliosphere. On the other side, more observations are required to describe the trends of the IPH velocity. SWAN made some spectral measurements between 2002 and 2007, so their analysis should increase significantly the current data set of IPH velocity measurements. This data set could also be extended with new $H S T /$ STIS observations to describe another waxing phase.

\section{CONCLUSION}

Updated analyses of $H S T$ /STIS observations provide IPH line-shifts of $22.6 \pm 0.5 \mathrm{~km} \mathrm{~s}^{-1}$ in $2001,23.5 \pm 0.5 \mathrm{~km} \mathrm{~s}^{-1}$ in 2012 , and $23.3 \pm 0.5 \mathrm{~km} \mathrm{~s}^{-1}$ in 2013 . These results have been compiled with results from $\mathrm{SOHO} / \mathrm{SWAN}$, and the whole data set has been compared over two solar cycles $(23 / 24)$ with predictions from a kinetic-fluid model of the heliosphere. This comparison shows that the LISM velocity has not changed since the Ulysses era. The discrepancy between the Ulysses and $I B E X$ results can be resolved by considering the upper value of the bounding range allowed by the equations used to derive the LISM velocity from IBEX data. None of the models can fit the whole data set of IPH velocity measurements, which call for additional modeling efforts but also for future observations to better characterize the trend induced by the solar cycle and the constraints due to the LISM.

This work was partly supported by the Space Telescope Science Institute, Program 22 of the Russian Academy of Sciences, the French Space Agency (CNES), Pierre and Marie Curie University (UPMC), and the French National Centre for Scientific Research (CNRS). Calculations of the hydrogen distributions were performed by using the supercomputers of the Lomonosov Moscow State University.

\section{REFERENCES}

Axford, W. I. 1972, in Solar Wind, ed. C. P. Sonett, P. J. Coleman, Jr., \& J. M. Wilcox (NASA SP-308; Washington, DC: Scientific and Technical Information Office, NASA), 609

Baranov, V. B., Krasnobaev, K. V., \& Kulikovskii, A. G. 1971, SPhD, 15, 791

Baranov, V. B., \& Malama, Y. G. 1993, JGR, 98, 15157

Ben-Jaffel, L., Puyoo, O., \& Ratkiewicz, R. 2000, ApJ, 533, 924

Ben-Jaffel, L., Strumik, M., Ratkiewicz, R., \& Grygorczuk, J. 2013, ApJ, 779,130

Bertaux, J. L., \& Blamont, J. E. 1971, A\&A, 11, 200

Bertin, P., Lallement, R., Ferlet, R., \& Vidal-Majar, A. 1993, JGR, 98, 15193 Blum, P. W., \& Fahr, H. J. 1970, A\&A, 4, 280

Bzowski, M., Fahr, H. J., Rucinski, D., \& Scherer, H. 1997, A\&A, 326, 396

Fahr, H. J., Grzedzielski, S., \& Ratkiewicz, R. 1988, AnGeo, 6, 337

Frisch, P. C., Redfield, S., \& Slavin, J. D. 2011, ARA\&A, 49, 237

Hernandez, S., et al. 2014, STIS Instrument Handbook, Version 13.0 (Baltimore, MD: STScI)

Izmodenov, V. 2009, SSRv, 143, 139

Izmodenov, V., Alexashov, D., \& Myasnikov, A. 2005, A\&A, 437, L35

Izmodenov, V., Malama, Y. G., \& Ruderman, M. S. 2008, AdSpR, 41, 318

Izmodenov, V., Malama, Y. G., Ruderman, M. S., et al. 2009, SSRv, 146, 329

Izmodenov, V. V., Katushkina, O. A., Quemerais, E., \& Bzowski, M. 2013, in Cross-Calibration of Past and Present Far UV Spectra of Solar System Objects and the Heliosphere, ISSI Scientific Report Series, ed. R. M. Bonnet, E. Quemerais, \& M. Snow (New York: Springer), 13, 7

Katushkina, O. A., \& Izmodenov, V. V. 2010, AstL, 36, 297

Katushkina, O. A., \& Izmodenov, V. V. 2011, AdSpR, 48, 1967

Krimigis, S. M., Mitchell, D. G., Roelof, E. C., Hsieh, K. C., \& McComas, D. J. 2009, Sci, 326, 971

Lallement, R., Quémerais, E., Bertaux, J. L., et al. 2005, Sci, 307, 1447 
Lallement, R., Quémerais, E., Koutroumpa, D., et al. 2010, in AIP Conf. Proc. 1216, 12th International Solar Winf Conf., ed. M. Maksimovic et al. (Melville, NY: AIP), 555

Linsky, J. L., \& Wood, B. E. 1996, ApJ, 463, 254

Markwardt, C. B. 2009, in ASP Conf. Ser. 411, Astronomical Data Analysis Software and Systems XVIII, ed. D. A. Bohlender, D. Durand, \& P. Dowler (San Francisco, CA: ASP), 251

McComas, D. J., Alexashov, D., Bzowski, M., et al. 2012, Sci, 336, 1291

McComas, D. J., Allegrini, F., Bochsler, P., et al. 2009, Sci, 326, 959

Opher, M., Richardson, J. D., Toth, G., \& Gombosi, T. I. 2009, SSRv, 143,43

Parker, E. N. 1961, ApJ, 134, 20

Pogorelov, N. V., Heerikhuisen, J., Zank, G. P., Mitchell, J. J., \& Cairns, I. H. 2009, AdSpR, 44, 1337

Pogorelov, N. V., \& Matsuda, T. 1998, JGR, 103, 237
Quémerais, E. 2000, A\&A, 358, 353

Quémerais, E., \& Izmodenov, V. 2002, A\&A, 396, 269

Quémerais, E., Lallement, R., Bertaux, J.-L., et al. 2006, A\&A, 455, 1135

Quémerais, E., Lallement, R., Ferron, S., et al. 2006, JGR, 111, 09114

Ratkiewicz, R., Barnes, A., Molvik, G. A., et al. 1998, A\&A, 335, 363

Ratkiewicz, R., \& Grygorczuk, J. 2008, GeoRL, 35, L23105

Redfield, S., \& Linksy, J. L. 2008, ApJ, 673, 283

Thomas, G. E., \& Krassa, R. F. 1971, A\&A, 11, 218

Vincent, F. E., Ben-Jaffel, L., \& Harris, W. M. 2011, ApJ, 738, 135

Wallis, M. 1975, Natur, 254, 202

Walsh, J. R., Goudfrooij, P., \& Malamuth, E. 2001, Instrument Science Report STIS 2001-02

Witte, M. 2004, A\&A, 426, 835

Woodgate, B. E., Kimble, R. A., Bowers, C. W., et al. 1998, PASP, 110,1183 